Article

\title{
Pricing of Arithmetic Asian Options under Stochastic Volatility Dynamics: Overcoming the Risks of High-Frequency Trading
}

\author{
Chih-Chen Hsu ${ }^{1}$, Chung-Gee Lin ${ }^{2,3, *}$ and Tsung-Jung Kuo ${ }^{2}$ \\ 1 Department of Business Administration, Soochow University, Taipei 100006, Taiwan; \\ stevenhsu0801@scu.edu.tw \\ 2 Department of Financial Engineering and Actuarial Mathematics, Soochow University, \\ Taipei 100006, Taiwan; kim0918kimo@yahoo.com.tw \\ 3 Taiwan/Pervasive Artificial Intelligence Research (PAIR) Labs, Taipei 106031, Taiwan \\ * Correspondence: cglin@scu.edu.tw
}

Received: 30 October 2020; Accepted: 18 December 2020; Published: 20 December 2020

check for updates

\begin{abstract}
This research extended the model developed by Hull and White by integrating Taylor-series expansion into the model for deriving approximate analytical solutions for stochastic volatility forward-starting Asian options. Numerical experiments were performed to compare the proposed model with the Monte Carlo model over numerous simulations and demonstrated that the developed model has a pricing accuracy greater than $99 \%$. Furthermore, the computation time was approximately $10^{-5} \mathrm{~s}$ for each simulation. The model's outstanding computational performance demonstrates its capability to address the challenges of high-frequency trading.
\end{abstract}

Keywords: Asian option; Taylor series expansion technique; forward starting; high-frequency trading; stochastic volatility

\section{Introduction}

High-frequency trading (HFT) is a method of automated trading that involves using computer programs to rapidly perform large numbers of transactions. HFT is controversial and has received some harsh criticism [1-3]. The qualitative changes in the functioning principles and structure of the derivatives market have a substantial impact on processes associated with investment activities so much so that major financial markets are regulated by policy measures that aim to reduce the threat from HFT. This evolution has already led to changes in how information is disseminated to traders and has given rise to new forms of trading. Therefore, a set of faultless regulations and hedging tools is necessary to restrict the disturbance from HFT, avoid circuit breakers, and maintain global financial order.

G20 leaders have aimed to avoid disturbances in interest rates and the foreign exchange derivatives market by committing to increasing transparency in derivatives markets through the implementation of mandatory reporting of derivatives contracts [4-6]. To address regulators' concerns and the potential threats of HFT, a forward-starting Asian option (FSAO) evaluation formula was developed in this study to cope with the potential instability of interest rates, equity, and commodity and foreign-exchange markets. The FSAO can be regarded as a special type of Asian option in which the arithmetic average price is calculated from the first trading date of the contract.

The advantages of the Asian option are manifold. First, it is popular in practice in the financial market because it has a lower cost than its counterpart, the plain vanilla option. Second, averaging underlying asset prices can prevent manipulation from the financial market [7-9]. Third, it is commonly 
used in interest rate and foreign exchange markets, providing an expiry price or strike price that remains more stable than plain vanilla over contract periods.

In academia, an FSAO is a path-dependent derivative; its payoff function depends on the average value of the underlying asset over a relatively short time period in which the option has reached partial maturity and is near its expiration date. A precise analytical solution does not exist. Thus, extensive research has investigated such options using numerical approaches. This paper briefly catalogues four main methods described in the literature: The Monte Carlo approach with variance reduction, approximation of the unknown distribution on the average price with a more tractable one, derivation of the exact representation of the Asian option price, and extensions of the Heston model [10].

The first approach, Monte Carlo simulation with variance reduction, is a benchmark method for option valuation. It has often been employed directly for option pricing or to assist in solving complex partial differential equations in option valuations. Well-known examples of the direct application of Monte Carlo simulation for option evaluation are in the studies by [11,12]. They numerically solved pricing partial differential equations, which inspired a series of subsequent studies, such as those by [13-18]. The main drawback of this evaluation approach is that it lacks computational efficiency.

In the second approach, the unknown distribution of the Asian option is approximated to obtain an approximate analytical option price formula. Well-known examples of researchers who have adopted this approach are [19-28]. Generally, the method involves applying Edgeworth expansion or a Taylor series approach to a log-normal reference distribution to approximate the distribution of the arithmetic average of the underlying price or strike price. For example, [29] used a moment-matched compound gamma distribution. These researchers developed analytic option price formulas, but the formulas lack reliable error estimates.

Introduced by [30], the third approach involves the use of the reciprocal of the average, for which the Laguerre series converges. Dufresne improved non-closed form solutions by using an advance mathematical technique. [31] adopted this approach to derive an analytical solution for complex option evaluations. However, this approach does not guarantee that the series will converge to the true price and does not consider the stochastic volatility effect.

First developed by [10], the Heston model has attracted considerable attention, with extensive research exploring its explicit (distributional and pathwise) solutions and exact Monte Carlo simulation. For example, [32] studied the exact simulation of stochastic volatility and other affine jump diffusion processes, and [33] explored explicit Heston solutions and stochastic approximation for path-dependent option pricing. Additionally, [34] recently developed exact option pricing methods for the popular $3 / 2$ model. Finally, an exotic unified approach to pricing Asian options in stochastic jump diffusion models, which was extended to include alternative exotic contracts by [35].

Motivated by these empirical results, the present study extended the research of Hull and White $[19,20,25,28]$ to include a Taylor series expansion technique for deriving the approximate analytical solution of an FSAO in the context of stochastic volatility pricing.

This study contributes to the literature by providing an efficient and accurate analytical solution for a stochastic-volatility FSAO (SVFSAO). Empirical evidence strongly supports the use of two well-separated timescales during evaluation.

The remainder of this paper is structured as follows. Section 2 outlines the problem being addressed, describes the derivation of an approximate analytic SVFSAO with Taylor expansion, and presents the solution for the optimal hedge ratio. Section 3 discusses the model's pricing and hedging performance in comparison with the solutions of the Monte Carlo simulation, which served as the benchmark. Section 4 presents conclusions.

\section{Materials and Methods}

This paper first provides the approximate analytical solution for the SVFSAO (Because of the complexity of deriving the SVFSAO, the whole derivation process is not included in this paper. Readers who are interested in the aforementioned derivation may receive more details from the authors upon 
reasonable request.) and subsequently addresses the hedge ratio of the SVFSAO. Details regarding the derivation process of the approximate analytical solution for the hedge ratio are provided in the Appendix A.

\subsection{Approximate Analytical Solution for SVFSAO Pricing}

Following Hull and White [1], Tsao, Chang, and Lin [14], and Lin and Chang [28], a European-style FSAO with stochastic volatility written on an asset with maturity date $T$ was investigated.

The price of the underlying asset, which was tradable and uncorrelated with aggregate consumption, was assumed to follow geometric Brownian motion in a risk-neutral world with stochastic volatility and was calculated as follows:

$$
\begin{gathered}
\frac{d S_{t}}{S_{t}}=(r-d) d t+\sigma_{t} d Z_{t}, \\
\frac{d \sigma_{t}{ }^{2}}{\sigma_{t}{ }^{2}}=\mu d t+\xi d W_{t},
\end{gathered}
$$

where $S_{t}$ is the underlying asset price at time $t, r$ is the annualized risk-free interest rate, $d$ is the continuous yield, $\sigma_{t}$ is the instantaneous volatility of the underlying asset at time $t, \mu$ is the drift term, and $\xi$ is the volatility term for the variance of the underlying asset. Both $\mu$ and $\xi$ remain constant over time. In addition, $d Z_{t}$ and $d W_{t}$ are independent Wiener processes.

Following the settings of Tsao, Chang, and Lin [14], this paper assumes that the strike price $K$ of option is the arithmetic average of underlying asset prices over the period $[T-A, T]$. It follows that the strike price $K$ is

$$
K=\frac{1}{A} \int_{T-A}^{T} S_{u} d u
$$

where $T-A$ is the time instant after time zero of the option's issuance. If $T-A=0$, then this option becoming a plain vanilla Asian option. It is important to distinguish the following time windows: $[0, T-A]$ and $[T-A, T]$. Before $T-A$, investors know nothing about the exercise price $K$. After time $T-A$ information about $K$ gradually accumulates.

\section{Time Window before $T-A$}

The price of a forward starting Asian call option at time $t(0<t \leq T-A)$ is denoted as

$$
C=e^{-r(T-t)} E\left[\left(S_{T}-K\right)^{+} \mid \mathcal{F}_{t}\right]
$$

where $E$ is the conditional expectation operator with respect to the risk-neutral probability measure and $\mathcal{F}_{t}$ is a filtration.

From Tsao, Chang, and Lin [14], it is straightforward to have the analytic approximation formula for forward starting Asian call option as

$$
C=S_{t} e^{-(r-d) A-d(T-t)}\left[m_{1} N\left(\frac{m_{1}}{\sqrt{v_{1}}}\right)+\sqrt{\frac{v_{1}}{2 \pi}} e^{\frac{-m_{1}^{2}}{2 v_{1}}}\right]
$$

where

$$
\begin{gathered}
m_{1}=\frac{\left(r-d-\frac{\sigma^{2}}{2}\right) A}{2}+\frac{A \sigma^{2}}{4}+\frac{\left(r-d-\frac{\sigma^{2}}{2}\right)^{2} A^{2}}{3} \\
v_{1}=\frac{A \sigma^{2}}{3}+\frac{A^{2} \sigma^{2}}{4}+\frac{7\left(r-d-\frac{\sigma^{2}}{2}\right)^{2} A^{3} \sigma^{2}}{15}+\frac{3\left(r-d-\frac{\sigma^{2}}{2}\right) A^{2} \sigma^{2}}{4}
\end{gathered}
$$

Time Window after $T-A$ 
For the case of time $t(T-A<t \leq T)$, at time $t$, the investors knew the information of the underlying asset price between $\left[S_{T-A}, S_{t}\right]$. The strike price can be split into two parts as follows,

$$
K=M_{t}+\frac{1}{A} \int_{t}^{T} S_{u} d u
$$

where

$$
M_{t}=\frac{1}{A} \int_{T-A}^{t} S_{u} d u
$$

The analytic approximation formula for forward starting Asian call option is straightforward as

$$
C=S_{t} e^{-r(T-t)}\left[m_{2} N\left(\frac{m_{2}}{\sqrt{v_{2}}}\right)+\sqrt{\frac{v_{2}}{2 \pi}} e^{\frac{-m_{2}{ }^{2}}{2 v_{2}}}\right]
$$

where

$$
\begin{aligned}
& m_{2}=1+(r-\left.d-\frac{\sigma^{2}}{2}\right)(T-t)-\frac{(T-t)}{A}-\frac{\left(r-d-\frac{\sigma^{2}}{2}\right)(T-t)^{2}}{2 A}-\frac{M_{t}}{S_{t}}+\frac{(T-t) \sigma^{2}}{2} \\
&+(T-t)^{2}\left(\frac{\sigma\left(r-d-\frac{\sigma^{2}}{2}\right)^{2}}{2}-\frac{\sigma^{2}}{4 A}\right)-(T-t)^{3} \frac{\sigma\left(r-d-\frac{\sigma^{2}}{2}\right)^{2}}{6 A} \\
& v_{2}=(T-t) \sigma^{2}\left[1-\frac{(T-t)}{A}+\frac{(T-t)^{2}}{3 A^{2}}\right]+\sigma^{2}(T-t)^{2}\left[2\left(r-d-\frac{\sigma^{2}}{2}\right)+\frac{\sigma^{2}}{2}\right] \\
&+ \sigma^{2}(T-t)^{3}\left[\left(r-d-\frac{\sigma^{2}}{2}\right)^{2}-\frac{\sigma^{2}}{3 A}-\frac{5\left(r-d-\frac{\sigma^{2}}{2}\right)}{3 A}\right] \\
&+ \sigma^{2}(T-t)^{4}\left[\frac{\sigma^{2}}{12 A^{2}-\frac{\sigma^{2}}{2}\left(r-d-\frac{\sigma^{2}}{2}\right)^{2}}+\frac{5\left(r-d-\frac{\sigma^{2}}{2}\right)}{3 A}\right] \\
&+ \sigma^{2}(T-t)^{5} \frac{2\left(r-d-\frac{\sigma^{2}}{2}\right)^{2}}{15 A^{2}}
\end{aligned}
$$

Let $\overline{\mathrm{V}}$ be the mean variance over the time interval $[0, T]$, from Hull and White [1]:

$$
\overline{\mathrm{V}}=\frac{1}{T} \int_{0}^{T} \sigma_{u}^{2} d u
$$

The first three moments of $\overline{\mathrm{V}}$ at $\mu=0$ can be derived as (Following the settings of Hull and White [19], Lin, and Chang [28], and to simplify the derivation of the analytic approximate solution for options, this paper only discusses the case in which $\mu=0$. However, the case in which $\mu \neq 0$ and the moments of $\bar{V}$ with nonzero $\mu$ can be derived without considerable effort; see Hull and White [19] for further details.)

$$
\begin{gathered}
E(\overline{\mathrm{V}})=V_{0}, \\
E\left(\overline{\mathrm{V}}^{2}\right)=\frac{2\left(e^{\xi^{2} T}-\xi^{2} T-1\right)}{\xi^{4} T^{2}} V_{0}^{2}, \text { and } \\
E\left(\overline{\mathrm{V}}^{3}\right)=\frac{\left(e^{3 \xi^{2} T}-9 e^{\xi^{2} T}-6 \xi^{2} T+8\right)}{3 \xi^{6} T^{3}} V_{0}^{3} .
\end{gathered}
$$

The derivations of variance and skewness of $\bar{V}$ are straightforward as,

$$
V(\overline{\mathrm{V}})=\frac{2 \sigma_{0}^{4}\left(e^{k}-k-1\right)}{k^{2}}-\sigma_{0}^{4} \text { and }
$$




$$
\operatorname{Skew}(\overline{\mathrm{V}})=\sigma_{0}^{6}\left[\frac{e^{3 k}-(9+18 k) e^{k}+\left(8+24 k+18 k^{2}+6 k^{3}\right)}{3 k^{3}}\right],
$$

where $k=\xi^{2} T$.

Proposition 1. The approximate analytical solution $f\left(S_{0}, \sigma_{0}^{2}\right)$ for pricing the SVFSAO when $t \in[0, T-A, T]$ can be approximated using the following formula:

$$
\begin{aligned}
f\left(S_{0}, \sigma_{0}^{2}\right) & =S_{t} e^{-(r-d) A-d(T-t)}\left[m_{1} N\left(\frac{m_{1}}{\sqrt{v_{1}}}\right)+\sqrt{\frac{v_{1}}{2 \pi}} e^{-\frac{m_{1}^{2}}{2 v_{1}}}\right] \\
& +\frac{1}{2} S_{t} e^{-(r-d) A-d(T-t)}\left[M_{12} N\left(\frac{m_{1}}{\sqrt{v_{1}}}\right)+\frac{1}{\sqrt{2 \pi}} e^{-\frac{m_{1}^{2}}{2 v_{1}}} M_{11} \bar{A}_{1}-\frac{1}{\sqrt{32 \pi}} e^{-\frac{m_{1}^{2}}{2 v_{1}}} v_{1}^{-\frac{3}{2}} V_{11}^{2}\right. \\
& \left.+\frac{1}{\sqrt{8 \pi}} e^{-\frac{m_{1}^{2}}{2 v_{1}}} v_{1}^{-\frac{1}{2}} V_{11} \bar{B}_{1}+\frac{1}{\sqrt{8 \pi}} e^{-\frac{m_{1}^{2}}{2 v_{1}}} v_{1}^{-\frac{1}{2}} V_{12}\right] \times\left[\frac{2 \sigma^{4}\left(e^{k}-k-1\right)}{k^{2}}-\sigma^{4}\right] \\
& +\frac{1}{6} S_{t} e^{-(r-d) A-d(T-t)}\left[M_{13} N\left(\frac{m_{1}}{\sqrt{v_{1}}}\right)+\frac{1}{\sqrt{2 \pi}} e^{-\frac{m_{1}^{2}}{2 v_{1}}} \alpha_{11}+\frac{1}{\sqrt{32 \pi}} e^{-\frac{m_{1}^{2}}{2 v_{1}}} \alpha_{12}\right. \\
+ & \left.\frac{1}{\sqrt{8 \pi}} e^{-\frac{m_{1}^{2}}{2 v_{1}}}\left(\alpha_{13}+\alpha_{14}\right)\right] \times \sigma^{6}\left[\frac{e^{3 k}-(9+18 k) e^{k}+\left(8+24 k+18 k^{2}-6 k^{3}\right)}{3 k^{3}}\right]
\end{aligned}
$$

where

$$
\begin{aligned}
& \bar{A}_{1}=\frac{\partial}{\partial \bar{V}}\left(\frac{m_{1}}{\sqrt{v_{1}}}\right)=M_{11} v_{1}^{-\frac{1}{2}}-\frac{1}{2} m_{1} v_{1}^{-\frac{3}{2}} V_{11} \\
& \bar{B}_{1}=\frac{\partial}{\partial \bar{V}}\left(-\frac{m_{1}^{2}}{2 v_{1}}\right)=-m_{1} v_{1}^{-1} M_{11}+\frac{1}{2} m_{1}^{2} v_{1}^{-2} V_{11} \\
& \alpha_{11}=2 M_{12} \bar{A}_{1}+M_{11} \bar{A}_{1} \bar{B}_{1}+M_{11}\left(v_{1}^{-\frac{1}{2}} M_{12}-v_{1}^{-\frac{3}{2}} M_{11} V_{11}-\frac{1}{2} m_{1} v_{1}^{-\frac{3}{2}} V_{12}+\frac{3}{4} m_{1} v_{1}^{-\frac{5}{2}} V_{11}^{2}\right) \\
& \alpha_{12}=\frac{3}{2} v_{1}^{-\frac{5}{2}} V_{11}^{3}-v_{1}^{-\frac{3}{2}} V_{11}^{2} \bar{B}_{1}-2 v_{1}^{-\frac{3}{2}} V_{11} V_{12} \\
& \alpha_{13}=-\frac{1}{2} v_{1}^{-\frac{3}{2}} V_{11} V_{12}+v_{1}^{-\frac{1}{2}} V_{12} \bar{B}_{1}+v_{1}^{-\frac{1}{2}} V_{13} \\
& \alpha_{14}=-\frac{1}{2} v_{1}^{-\frac{3}{2}} V_{11}^{2} \bar{B}_{1}+v_{1}^{-\frac{1}{2}} V_{11} \bar{B}_{1}^{2}+v_{1}^{-\frac{1}{2}} V_{12} \bar{B}_{1} \\
& +v_{1}^{-\frac{1}{2}} V_{11}\left(-v_{1}^{-1} M_{11}^{2}+2 m_{1} v_{1}^{-2} M_{11} V_{11}-m_{1} v_{1}^{-1} M_{12}-m_{1}^{2} v_{1}^{-3} V_{11}^{2}+\frac{1}{2} m_{1}^{2} v_{1}^{-2} V_{12}\right) \\
& M_{11}=\frac{\partial m_{1}}{\partial \bar{V}}=-\frac{1}{3} A^{2}(r-d)+\frac{1}{6} A^{2} \bar{V} \\
& M_{12}=\frac{\partial^{2} m_{1}}{\partial \bar{V}^{2}}=\frac{1}{6} A^{2} \\
& M_{13}=\frac{\partial^{3} m_{1}}{\partial \bar{V}^{3}}=0 \\
& V_{11}=\frac{\partial v_{1}}{\partial \bar{V}}=\left[\frac{1}{3} A+\frac{3}{4} A^{2}(r-d)+\frac{7}{15} A^{3}(r-d)^{2}\right]+\left[-\frac{1}{4} A^{2}-\frac{14}{15} A^{3}(r-d)\right] \bar{V}+\frac{7}{20} A^{3} \bar{V}^{2} \\
& V_{12}=\frac{\partial^{2} v_{1}}{\partial \bar{V}^{2}}=\left[-\frac{1}{4} A^{2}-\frac{14}{15} A^{3}(r-d)\right]+\frac{7}{10} A^{3} \bar{V} \\
& V_{13}=\frac{\partial^{3} v_{1}}{\partial \bar{V}^{3}}=\frac{7}{10} A^{3}
\end{aligned}
$$


When $t \in[0, T-A, T]$, then

$$
\begin{aligned}
f\left(S_{0}, \sigma_{0}^{2}\right) & =S_{t} e^{-r(T-t)}\left[m_{2} N\left(\frac{m_{2}}{\sqrt{v_{2}}}\right)+\sqrt{\frac{v_{2}}{2 \pi}} e^{-\frac{m_{2}^{2}}{2 v_{2}}}\right] \\
& +\frac{1}{2} S_{t} e^{-r(T-t)}\left[M_{22} N\left(\frac{m_{2}}{\sqrt{v_{2}}}\right)+\frac{1}{\sqrt{2 \pi}} e^{-\frac{m_{2}^{2}}{2 v_{2}}} M_{21} \bar{A}_{2}-\frac{1}{\sqrt{32 \pi}} e^{-\frac{m_{2}^{2}}{2 v_{2}}} v_{2}^{-\frac{3}{2}} V_{21}^{2}\right. \\
& \left.+\frac{1}{\sqrt{8 \pi}} e^{-\frac{m_{2}^{2}}{2 v_{2}}} v_{2}^{-\frac{1}{2}} V_{21} \bar{B}_{2}+\frac{1}{\sqrt{8 \pi}} e^{-\frac{m_{2}^{2}}{2 v_{2}}} v_{2}^{-\frac{1}{2}} V_{22}\right] \times\left[\frac{2 \sigma^{4}\left(e^{k}-k-1\right)}{k^{2}}-\sigma^{4}\right] \\
& +\frac{1}{6} S_{t} e^{-r(T-t)}\left[M_{23} N\left(\frac{m_{2}}{\sqrt{v_{2}}}\right)+\frac{1}{\sqrt{2 \pi}} e^{-\frac{m_{2}^{2}}{2 v_{2}}} \alpha_{21}+\frac{1}{\sqrt{32 \pi}} e^{-\frac{m_{2}^{2}}{2 v_{2}}} \alpha_{22}\right. \\
& \left.+\frac{1}{\sqrt{8 \pi}} e^{-\frac{m_{2}^{2}}{2 v_{2}}}\left(\alpha_{23}+\alpha_{24}\right)\right] \times \sigma^{6}\left[\frac{e^{3 k}-(9+18 k) e^{k}+\left(8+24 k+18 k^{2}-6 k^{3}\right)}{3 k^{3}}\right]
\end{aligned}
$$

where

$$
\begin{aligned}
& \bar{A}_{2}=\frac{\partial}{\partial \bar{V}}\left(\frac{m_{2}}{\sqrt{v_{2}}}\right)=M_{21} v_{2}^{-\frac{1}{2}}-\frac{1}{2} m_{2} v_{2}^{-\frac{3}{2}} V_{21} \\
& \bar{B}_{2}=\frac{\partial}{\partial \bar{V}}\left(-\frac{m_{2}^{2}}{2 v_{2}}\right)=-m_{2} v_{2}^{-1} M_{21}+\frac{1}{2} m_{2}^{2} v_{2}^{-2} V_{21} \\
& \alpha_{21}=2 M_{22} \bar{A}_{2}+M_{21} \bar{A}_{2} \bar{B}_{2}+M_{21}\left(v_{2}^{-\frac{1}{2}} M_{22}-v_{2}^{-\frac{3}{2}} M_{21} V_{21}-\frac{1}{2} m_{2} v_{2}^{-\frac{3}{2}} V_{22}+\frac{3}{4} m_{2} v_{2}^{-\frac{5}{2}} V_{21}^{2}\right) \\
& \alpha_{22}=\frac{3}{2} v_{2}^{-\frac{5}{2}} V_{21}^{3}-v_{2}^{-\frac{3}{2}} V_{21}^{2} \bar{B}_{2}-2 v_{2}^{-\frac{3}{2}} V_{21} V_{22} \\
& \alpha_{23}=-\frac{1}{2} v_{2}^{-\frac{3}{2}} V_{21} V_{22}+v_{2}^{-\frac{1}{2}} V_{22} \bar{B}_{2}+v_{2}^{-\frac{1}{2}} V_{23} \\
& \alpha_{24}=-\frac{1}{2} v_{2}^{-\frac{3}{2}} V_{21}^{2} \bar{B}_{2}+v_{2}^{-\frac{1}{2}} V_{21} \bar{B}_{2}^{2}+v_{2}^{-\frac{1}{2}} V_{22} \bar{B}_{2} \\
& +v_{2}^{-\frac{1}{2}} V_{21}\left(-v_{2}^{-1} M_{21}^{2}+2 m_{2} v_{2}^{-2} M_{21} V_{21}-m_{2} v_{2}^{-1} M_{22}-m_{2}^{2} v_{2}^{-3} V_{21}^{2}+\frac{1}{2} m_{2}^{2} v_{2}^{-2} V_{22}\right) \\
& M_{21}=\frac{\partial m_{2}}{\partial \bar{V}}=\left[-\frac{1}{2}(T-t)^{2}(r-d)+\frac{1}{6 A}(T-t)^{3}(r-d)\right]+\left[\frac{1}{4}(T-t)^{2}-\frac{1}{12 A}(T-t)^{3}\right] \bar{V} \\
& M_{22}=\frac{\partial^{2} m_{2}}{\partial \bar{V}^{2}}=\left[\frac{1}{4}(T-t)^{2}-\frac{1}{12 A}(T-t)^{3}\right] \\
& M_{23}=\frac{\partial^{3} m_{2}}{\partial \bar{V}^{3}}=0 \\
& V_{21}=\frac{\partial v_{2}}{\partial \bar{V}}=\left[1-\frac{1}{A}(T-t)+2(T-t)(r-d)+\frac{1}{3 A^{2}}(T-t)^{2}-\frac{5}{3 A}(T-t)^{2}(r-d)\right. \\
& +(T-t)^{2}(r-d)^{2}+\frac{5}{12 A^{2}}(T-t)^{3}(r-d)-\frac{2}{3 A}(T-t)^{3}(r-d)^{2} \\
& \left.+\frac{2}{15 A^{2}}(T-t)^{4}(r-d)^{2}\right](T-t)+\left[-1+\frac{1}{A}(T-t)-2(T-t)(r-d)\right. \\
& \left.-\frac{1}{4 A^{2}}(T-t)^{2}+\frac{4}{3 A}(T-t)^{2}(r-d)-\frac{4}{15 A^{2}}(T-t)^{3}(r-d)\right](T-t)^{2} \bar{V} \\
& +\left[\frac{3}{4}-\frac{1}{2 A}(T-t)+\frac{1}{10 A^{2}}(T-t)^{2}\right](T-t)^{3} \bar{V}^{2}
\end{aligned}
$$




$$
\begin{gathered}
V_{22}=\frac{\partial^{2} v_{2}}{\partial \bar{V}^{2}}=\left[-1+\frac{1}{A}(T-t)-2(T-t)(r-d)-\frac{1}{4 A^{2}}(T-t)^{2}+\frac{4}{3 A}(T-t)^{2}(r-d)\right. \\
\left.-\frac{4}{15 A^{2}}(T-t)^{3}(r-d)\right](T-t)^{2}+\left[\frac{3}{2}-\frac{1}{A}(T-t)+\frac{1}{5 A^{2}}(T-t)^{2}\right](T-t)^{3} \bar{V} \\
V_{23}=\frac{\partial^{3} v_{2}}{\partial \bar{V}^{3}}=\left[\frac{3}{2}-\frac{1}{A}(T-t)+\frac{1}{5 A^{2}}(T-t)^{2}\right](T-t)^{3}
\end{gathered}
$$

\subsection{Approximate Analytical Solution for Hedging the SVFSAO}

After the analytical solution for pricing the SVFSAO is derived, the hedge ratio $\left(\Delta=\frac{\partial f\left(S_{0}, \sigma_{0}^{2}\right)}{\partial S_{0}}\right)$ can be derived directly through differentiation of the option price $f\left(S_{0}, \sigma_{0}^{2}\right)$ with respect to the asset price $S_{0}$ at the present time:

$$
\text { Delta }=\Delta=\frac{\partial f\left(S_{0}, \sigma_{0}^{2}\right)}{\partial S_{0}}
$$

Proposition 2. The approximate analytical solution for hedging the SVFSAO at $\boldsymbol{t} \in[\mathbf{0}, \boldsymbol{T}-\boldsymbol{A}, \boldsymbol{T}]$ can be approximated using the following formula:

$$
\begin{aligned}
& \text { Delta }=\Delta=\frac{\partial f\left(S_{0}, \boldsymbol{\sigma}_{0}^{2}\right)}{\partial S_{0}}=e^{-(r-d) A-d(T-t)}\left[m_{1} N\left(\frac{m_{1}}{\sqrt{v_{1}}}\right)+\sqrt{\frac{v_{1}}{2 \pi}} e^{-\frac{m_{1}^{2}}{2 v_{1}}}\right] \\
& +\frac{1}{2} e^{-(r-d) A-d(T-t)}\left[\boldsymbol{M}_{12} N\left(\frac{m_{1}}{\sqrt{v_{1}}}\right)+\frac{1}{\sqrt{2 \pi}} e^{-\frac{m_{1}^{2}}{2 v_{1}}} \boldsymbol{M}_{11} \bar{A}_{1}-\frac{1}{\sqrt{32 \pi}} e^{-\frac{m_{1}^{2}}{2 v_{1}}} \boldsymbol{v}_{1}^{-\frac{3}{2}} V_{11}^{2}\right. \\
& \left.+\frac{1}{\sqrt{8 \pi}} e^{-\frac{m_{1}^{2}}{2 v_{1}}} \boldsymbol{v}_{1}^{-\frac{1}{2}} \boldsymbol{V}_{11} \bar{B}_{1}+\frac{1}{\sqrt{8 \pi}} e^{-\frac{m_{1}^{2}}{2 v_{1}}} \boldsymbol{v}_{1}^{-\frac{1}{2}} \mathbf{V}_{12}\right] \times\left[\frac{2 \boldsymbol{\sigma}^{4}\left(e^{k}-k-1\right)}{k^{2}}-\boldsymbol{\sigma}^{4}\right] \\
& +\frac{1}{6} e^{-(r-d) A-d(T-t)}\left[M_{13} N\left(\frac{m_{1}}{\sqrt{v_{1}}}\right)+\frac{1}{\sqrt{2 \pi}} e^{-\frac{m_{1}^{2}}{2 v_{1}}} \alpha_{11}+\frac{1}{\sqrt{32 \pi}} e^{-\frac{m_{1}^{2}}{2 v_{1}}} \alpha_{12}\right. \\
& \left.+\frac{1}{\sqrt{8 \pi}} e^{-\frac{m_{1}^{2}}{2 v_{1}}}\left(\boldsymbol{\alpha}_{13}+\boldsymbol{\alpha}_{14}\right)\right] \times \boldsymbol{\sigma}^{6}\left[\frac{e^{3 k}-(9+18 k) e^{k}+\left(8+24 k+18 k^{2}-6 k^{3}\right)}{3 k^{3}}\right]
\end{aligned}
$$

When $t \in[0, T-A, T]$, then 


$$
\begin{aligned}
& \text { Delta }=\Delta=\frac{\partial f\left(S_{0}, \boldsymbol{\sigma}_{0}^{2}\right)}{\partial S_{0}}=e^{-r(T-t)}\left[m_{2} N\left(\frac{m_{2}}{\sqrt{v_{2}}}\right)+\sqrt{\frac{v_{2}}{2 \pi}} e^{-\frac{m_{2}^{2}}{2 v_{2}}}+S_{t} N\left(\frac{m_{2}}{\sqrt{v_{2}}}\right) \frac{\partial m_{2}}{\partial S_{t}}\right] \\
& +\frac{1}{2}\left\{e ^ { - r ( T - t ) } \left[\boldsymbol{M}_{22} N\left(\frac{m_{2}}{\sqrt{v_{2}}}\right)+\frac{1}{\sqrt{2 \pi}} e^{-\frac{m_{2}^{2}}{2 v_{2}}} \boldsymbol{M}_{21} \bar{A}_{2}-\frac{1}{\sqrt{32 \pi}} e^{-\frac{m_{2}^{2}}{2 v_{2}}} \boldsymbol{v}_{2}^{-\frac{3}{2}} V_{21}^{2}\right.\right. \\
& \left.+\frac{1}{\sqrt{8 \pi}} e^{-\frac{m_{2}^{2}}{2 v_{2}}} \boldsymbol{v}_{2}^{-\frac{1}{2}} \boldsymbol{V}_{22}+\frac{1}{\sqrt{8 \pi}} e^{-\frac{m_{2}^{2}}{2 v_{2}}} \boldsymbol{v}_{2}^{-\frac{1}{2}} \boldsymbol{V}_{21} \overline{\boldsymbol{B}}_{2}\right]+S_{t} \boldsymbol{e}^{-r(T-t)}\left[\frac{1}{\sqrt{2 \pi}} e^{-\frac{m_{2}^{2}}{2 v_{2}}} \boldsymbol{M}_{22} \boldsymbol{v}_{2}^{-\frac{1}{2}} \frac{\partial m_{2}}{\partial S_{t}}\right. \\
& -\frac{1}{\sqrt{2 \pi}} e^{-\frac{m_{2}^{2}}{2 v_{2}}} \boldsymbol{m}_{2} \boldsymbol{v}_{2}^{-1} \boldsymbol{M}_{21} \bar{A}_{2} \frac{\partial m_{2}}{\partial S_{t}}+\frac{1}{\sqrt{32 \pi}} e^{-\frac{m_{2}^{2}}{2 v_{2}}} \boldsymbol{m}_{2} \boldsymbol{v}_{2}^{-\frac{5}{2}} V_{21}^{2} \frac{\partial m_{2}}{\partial S_{t}}-\frac{1}{\sqrt{8 \pi}} e^{-\frac{m_{2}^{2}}{2 v_{2}}} \boldsymbol{m}_{2} \boldsymbol{v}_{2}^{-\frac{3}{2}} V_{22} \frac{\partial m_{2}}{\partial S_{t}} \\
& \left.\left.-\frac{1}{\sqrt{8 \pi}} e^{-\frac{m_{2}^{2}}{2 v_{2}}} \boldsymbol{m}_{2} \boldsymbol{v}_{2}^{-\frac{3}{2}} \boldsymbol{V}_{21} \overline{\boldsymbol{B}}_{2} \frac{\partial m_{2}}{\partial S_{t}}+\frac{1}{\sqrt{2 \pi}} e^{-\frac{m_{2}^{2}}{2 v_{2}}} \boldsymbol{M}_{21} \frac{\partial \bar{A}_{2}}{\partial S_{t}}+\frac{1}{\sqrt{8 \pi}} e^{-\frac{m_{2}^{2}}{2 v_{2}}} \boldsymbol{v}_{2}^{-\frac{1}{2}} \boldsymbol{V}_{21} \frac{\partial \bar{B}_{2}}{\partial S_{t}}\right]\right\} \\
& \times\left[\frac{2 \sigma^{4}\left(e^{k}-k-1\right)}{k^{2}}-\sigma^{4}\right] \\
& +\frac{1}{6}\left\{e^{-r(T-t)}\left[M_{23} N\left(\frac{m_{2}}{\sqrt{v_{2}}}\right)+\frac{1}{\sqrt{2 \pi}} e^{-\frac{m_{2}^{2}}{2 v_{2}}} \alpha_{21}+\frac{1}{\sqrt{32 \pi}} e^{-\frac{m_{2}^{2}}{2 v_{2}}} \alpha_{22}+\frac{1}{\sqrt{8 \pi}} e^{-\frac{m_{2}^{2}}{2 v_{2}}}\left(\alpha_{23}+\alpha_{24}\right)\right]\right. \\
& +\boldsymbol{S}_{t} e^{-r(T-t)}\left[\frac{1}{\sqrt{2 \pi}} e^{-\frac{m_{2}^{2}}{2 v_{2}}} \boldsymbol{M}_{23} \boldsymbol{v}_{2}^{-\frac{1}{2}} \frac{\partial m_{2}}{\partial S_{t}}-\frac{1}{\sqrt{2 \pi}} e^{-\frac{m_{2}^{2}}{2 v_{2}}} \boldsymbol{m}_{2} \boldsymbol{v}_{2}^{-1} \alpha_{21} \frac{\partial m_{2}}{\partial S_{t}}+\frac{1}{\sqrt{2 \pi}} e^{-\frac{m_{2}^{2}}{2 v_{2}}} \frac{\partial \alpha_{21}}{\partial S_{t}}\right. \\
& -\frac{1}{\sqrt{32 \pi}} e^{-\frac{m_{2}^{2}}{2 v_{2}}} m_{2} v_{2}^{-1} \alpha_{22} \frac{\partial m_{2}}{\partial S_{t}}+\frac{1}{\sqrt{32 \pi}} e^{-\frac{m_{2}^{2}}{2 v_{2}} \frac{\partial \alpha_{22}}{\partial S_{t}}-\frac{1}{\sqrt{8 \pi}} e^{-\frac{m_{2}^{2}}{2 v_{2}}} m_{2} v_{2}^{-1}\left(\alpha_{23}+\alpha_{24}\right) \frac{\partial m_{2}}{\partial S_{t}}} \\
& +\frac{1}{\sqrt{8 \pi}} e^{\left.\left.-\frac{m_{2}^{2}}{2 v_{2}}\left(\frac{\partial \alpha_{23}}{\partial S_{t}}+\frac{\partial \alpha_{24}}{\partial S_{t}}\right)\right]\right\} \times \sigma^{6}\left[\frac{e^{3 k}-(9+18 k) e^{k}+\left(8+24 k+18 k^{2}-6 k^{3}\right)}{3 k^{3}}\right]}
\end{aligned}
$$

Please refer to the Appendix A for notations and proofs of the propositions.

\section{Results}

Monte Carlo simulation was performed to clarify the differences in pricing between a constant-volatility FSAO and SVFSAO according to the pricing results and computer operation time. Next, the accuracy of the approximate analytical solution for the SVFSAO was compared with the solution of Monte Carlo simulation, which served as the benchmark. Finally, the hedge ratio of the SVFSAO was determined and compared with that of a constant-volatility FSAO. The results of the aforementioned three numerical analyses demonstrate the high value of the developed model and its ability to address the potential risk incurred by HFT.

\subsection{Differences in Pricing between Constant-Volatility FSAOs and SVFSAOs}

To demonstrate the value of the developed SVFSAO, Monte Carlo simulation was performed to price FSAOs under the conditions of constant volatility and stochastic volatility in different scenarios (To ensure consistency with reality, for numerical investigations, it was assumed that there are 365 days in a year.).

As indicated in Table 1, pricing differences between FSAOs under constant volatility and stochastic volatility conditions were evident, exceeding $4 \%$ for several conditions. This result demonstrates that the constant-volatility FSAO pricing model could be prone to mispricing and have detrimental effects on users' justifications for hedging. 
Table 1. Comparison of FSAOs with stochastic and constant volatilities.

\begin{tabular}{|c|c|c|c|c|c|c|}
\hline & & (1) & (2) & (3) & (4) & (5) \\
\hline$\mu$ & $\xi$ & MC(SV) & $\mathrm{MC}(\mathrm{CV})$ & $\begin{array}{l}\text { Diff Ratio } \\
(2)-(1) /(2)\end{array}$ & $\begin{array}{l}\text { Time } \\
\text { (SV) }\end{array}$ & $\begin{array}{l}\text { Time } \\
\text { (CV) }\end{array}$ \\
\hline \multirow{3}{*}{0} & 0.3 & $\begin{array}{c}4.96240 \\
(0.02769)\end{array}$ & $\begin{array}{c}4.98923 \\
(0.02047)\end{array}$ & $0.537758 \%$ & 28.83718 & 11.98062 \\
\hline & 0.6 & $\begin{array}{c}4.88633 \\
(0.02786)\end{array}$ & $\begin{array}{c}4.98923 \\
(0.02047)\end{array}$ & $2.062443 \%$ & 28.67936 & 11.97470 \\
\hline & 0.9 & $\begin{array}{l}4.76376 \\
(0.028)\end{array}$ & $\begin{array}{c}4.98923 \\
(0.02047)\end{array}$ & $4.519134 \%$ & 28.41344 & 11.96720 \\
\hline \multirow{3}{*}{0.1} & 0.3 & $\begin{array}{c}5.08854 \\
(0.02862)\end{array}$ & $\begin{array}{c}4.98923 \\
(0.02047)\end{array}$ & $-1.990488 \%$ & 28.78062 & 11.96250 \\
\hline & 0.6 & $\begin{array}{c}5.00971 \\
(0.02881)\end{array}$ & $\begin{array}{c}4.98923 \\
(0.02047)\end{array}$ & $-0.410484 \%$ & 28.68312 & 11.96968 \\
\hline & 0.9 & $\begin{array}{c}4.88268 \\
(0.02895)\end{array}$ & $\begin{array}{c}4.98923 \\
(0.02047)\end{array}$ & $2.135600 \%$ & 28.41344 & 11.96500 \\
\hline \multirow{3}{*}{0.2} & 0.3 & $\begin{array}{l}5.21888 \\
(0.0296)\end{array}$ & $\begin{array}{c}4.98923 \\
(0.02047)\end{array}$ & $-4.602915 \%$ & 28.76970 & 11.96500 \\
\hline & 0.6 & $\begin{array}{c}5.13721 \\
(0.02978)\end{array}$ & $\begin{array}{c}4.98923 \\
(0.02047)\end{array}$ & $-2.965989 \%$ & 28.68812 & 11.96812 \\
\hline & 0.9 & $\begin{array}{c}5.00558 \\
(0.02994)\end{array}$ & $\begin{array}{c}4.98923 \\
(0.02047)\end{array}$ & $-0.327706 \%$ & 28.41032 & 11.95906 \\
\hline
\end{tabular}

Note: The parameters were set as follows: $S_{0}=100, r=10 \%, d=0 \%, \sigma_{0}=30 \%, T=180$ days, $A=90$ days. Option prices in columns (1) and (2) reflect stochastic and constant volatility conditions, respectively, of Monte Carlo simulation with 100,000 paths and 50 repetitions. The standard error is presented in parentheses. Column (3) displays the difference ratio, which denotes the percentage difference in pricing. Columns (4) and (5) respectively indicate the time required for simulation under stochastic and constant volatility conditions. Monte Carlo simulation was performed using a personal computer with the following specifications: P4-3.4 GHz with $1 \mathrm{~GB}$ RAM.

Computation time is a crucial factor for managing computer-based trading, especially HFT. The time required for computing a model price for options is at least $11 \mathrm{~s}$ for the case of a constant-volatility FSAO and at least $28 \mathrm{~s}$ for the case of an SVFSAO. A pricing technique that relies on simulation is incapable of handling HFT. In the present study, this numerical analysis supported the development of an approximate analytical solution for FSAOs.

Table 2 details the pricing performance of the present study's approximate analytical path-dependent FSAO solutions. Compared with Monte Carlo simulation, for a large sample path, the developed model had a superior pricing speed and accuracy, proving that the FSAO can be applied to manage HFT.

The values in the "AAS" column of Table 2 (The parameter settings were selected with reference to [25]) are the approximate analytical solutions for the SVFSAO. Compared with the solutions of Monte Carlo simulation, the present study's approximate analytical solutions were closer to the theoretical value, and all pricing errors were less than $1 \%$. Furthermore, the computing time for the approximate analytical solutions was more than 2 million times faster than that of the Monte Carlo solutions; only approximately $20 \mu$ s was required for computing a SVFSAO pricing solution. This computing performance is sufficient for dealing with the fast liquidity of HFT. 
Table 2. Comparison of the analytical solutions of the proposed FSAO and the Monte Carlo method.

\begin{tabular}{|c|c|c|c|c|c|c|}
\hline & (1) & (2) & (3) & (4) & (5) & (6) \\
\hline $\begin{array}{c}\mathrm{T} \\
\text { (Days) }\end{array}$ & MC & ASC & $\begin{array}{c}\text { Diff Ratio } \\
(2)-(1) /(1)\end{array}$ & $\begin{array}{l}\text { Time } \\
\text { (MC) }\end{array}$ & $\begin{array}{l}\text { Time } \\
\text { (AAS) }\end{array}$ & Efficiency $=(4) /(5)$ \\
\hline 30 & $\begin{array}{l}1.83410 \\
(0.0097)\end{array}$ & 1.837416 & $0.180797 \%$ & 57.28814 & $2.343 \times 10^{-5}$ & $2.445 \times 10^{6}$ \\
\hline 90 & $\begin{array}{c}3.36440 \\
(0.018)\end{array}$ & 3.377293 & $0.383218 \%$ & 57.51626 & $2.281 \times 10^{-5}$ & $2.522 \times 10^{6}$ \\
\hline 120 & $\begin{array}{c}3.96357 \\
(0.02133)\end{array}$ & 3.981609 & $0.455120 \%$ & 57.48656 & $2.312 \times 10^{-5}$ & $2.486 \times 10^{6}$ \\
\hline 240 & $\begin{array}{c}5.94518 \\
(0.03275)\end{array}$ & 5.980835 & $0.599730 \%$ & 57.19782 & $2.500 \times 10^{-5}$ & $2.288 \times 10^{6}$ \\
\hline
\end{tabular}

Note: The parameters were set as follows: $S_{0}=100, r=10 \%, d=0 \%, \mu=0, \xi=0.15, \sigma_{0}=30 \%, A=T / 2$ trading days. Column (1) is the results of the Monte Carlo (MC) method, with 100,000 paths and 50 repetitions. The standard error is presented in parenthesis. Column (2) displays the option value obtained using the analytical solution proposed in this study. Column (3) presents the difference ratio, which denotes the pricing difference as a percentage. Columns (4) and (5) indicate the time required for simulation using the MC method and the approximate analytical solution (AAS), respectively. Column (6) presents the efficiency (the required simulation time for the benchmark method divided by that required for the present study's formula). MC simulation was performed on a personal computer with the following specifications: P4-3.4 GHz with 1 GB RAM.

\subsection{Comparison of the Delta Value of FSAO between Constant and Stochastic Volatility Conditions}

Hedging is vital for option market participants. Without rebalancing the underlying asset position according to the hedging ratio, delta, option market participants cannot maintain a risk-free portfolio of options and underlying assets and may incur investment loss from the pricing changes of underlying assets.

Table 3 indicates the hedging performance in terms of the delta value under the developed SVFSAO and constant-volatility FSAO. The employed constant-volatility FSAO hedge model refers to the model developed by Tsao, Chang, and Lin [14].

Table 3. Comparison of the delta value of the FSAO between constant and stochastic volatility conditions.

\begin{tabular}{ccccc}
\hline & & $\mathbf{( 1 )}$ & $\mathbf{( 2 )}$ & $\mathbf{( 3 )}$ \\
\hline $\boldsymbol{\sigma}$ & $\boldsymbol{\xi}$ & $\begin{array}{c}\text { Delta } \\
\mathbf{( C V )}\end{array}$ & $\begin{array}{c}\text { Delta } \\
\mathbf{( S V )}\end{array}$ & $\begin{array}{c}\text { Diff Ratio } \\
\mathbf{( 2 )}-\mathbf{( 1 ) / ( 1 )}\end{array}$ \\
\hline \multirow{6}{*}{0.2} & 0.2 & $1.4381 \times 10^{-19}$ & $3.4619 \times 10^{-19}$ & $140.7296 \%$ \\
& 0.4 & $1.4381 \times 10^{-19}$ & $1.1246 \times 10^{-18}$ & $682.0450 \%$ \\
& 0.6 & $1.4381 \times 10^{-19}$ & $3.0113 \times 10^{-18}$ & $1993.9933 \%$ \\
& 0.8 & $1.4381 \times 10^{-19}$ & $6.9566 \times 10^{-18}$ & $4737.4300 \%$ \\
0.3 & 0.2 & $1.0431 \times 10^{-9}$ & $1.3115 \times 10^{-9}$ & $25.7356 \%$ \\
& 0.4 & $1.0431 \times 10^{-9}$ & $2.2116 \times 10^{-9}$ & $112.0294 \%$ \\
& 0.6 & $1.0431 \times 10^{-9}$ & $4.0376 \times 10^{-9}$ & $287.0919 \%$ \\
& 0.8 & $1.0431 \times 10^{-9}$ & $7.3144 \times 10^{-9}$ & $601.2435 \%$ \\
& 0.2 & $3.3847 \times 10^{-6}$ & $3.6421 \times 10^{-6}$ & $7.6060 \%$ \\
& 0.4 & $3.3847 \times 10^{-6}$ & $4.4579 \times 10^{-6}$ & $31.7077 \%$ \\
& 0.6 & $3.3847 \times 10^{-6}$ & $5.9667 \times 10^{-6}$ & $76.2859 \%$ \\
& 0.8 & $3.3847 \times 10^{-6}$ & $8.4084 \times 10^{-6}$ & $148.4258 \%$ \\
& 0.2 & $1.5510 \times 10^{-4}$ & $1.5954 \times 10^{-4}$ & $2.8660 \%$ \\
& 0.4 & $1.5510 \times 10^{-4}$ & $1.7323 \times 10^{-4}$ & $11.6924 \%$ \\
& 0.6 & $1.5510 \times 10^{-4}$ & $1.9726 \times 10^{-4}$ & $27.1859 \%$ \\
& 0.8 & $1.5510 \times 10^{-4}$ & $2.3357 \times 10^{-4}$ & $50.5983 \%$ \\
\hline
\end{tabular}

Note: The parameters were set as follows: $S_{0}=100, r=10 \%, d=0 \%, \mu=0, T=0.5, A=T / 2, t=0.75 T$. Column (1) presents the delta value of the call option when the analytical solution of the TCL model is used. Column (2) presents the delta value of the call option when the analytical solution of the proposed model is used. Column (3) provides the difference ratio, which denotes the delta difference as a percentage. 
The values in the "delta (SV)" column represent the developed model, which exhibited greater volatility than the values in the "delta (CV)" column for the TCL model. The differences between SV and $\mathrm{CV}$ were more obvious at higher $\xi$ values. The results in this table support that the constant-volatility FSAO model may underprice the hedge ratio delta. The market participants with FSAOs should be careful when using the TCL constant-volatility model because it could lead them to take the wrong hedging position.

The accuracy of the present study's pricing formula is based on two implications. The first is that the analytical solutions of hedge ratios for these options are reliable. The second is that the present study's pricing formula can be used as a control variable to enhance computational accuracy and efficiency when numerical methods (e.g., Monte Carlo simulation or binomial tree approaches) are being used for pricing American SVFSAOs. Pricing and hedging performance with respect to computation time and pricing accuracy are critical for handling the added risk introduced by the increase in computer-based trading, particularly HFT.

\section{Conclusions}

An approximate analytical solution for a stochastic-volatility Asian option valuation model based on the model introduced by Hull and White [1] that integrates Taylor series expansion was developed in this study. Greek hedging was also developed to verify the natural hedging ability of derivatives.

Numerical experiments demonstrated that the present study's approximate analytical solution outperforms performance and a higher computational efficiency compared with a benchmark approach for large-sample simulations. The approximate analytical solution in this study can serve as a practical tool for pricing and hedging SVFSAOs; it is straightforward to implement and is suitable for other complex derivative pricing because of the importance of computational speed. Thus, this research provides evidence that the developed SVFSAO model is capable of overcoming the potential challenges, such as liquidity and volatility toxicity, of HFT.

Other advantages of the developed model are summarized as follows: First, it can easily calculate the hedge ratios of SVFSAOs for use in hedging SVFSAO valuations. Second, the developed FSAO valuation model can be applied as a generalized Asian option valuation model under the condition of stochastic volatility pricing. The developed SVFSAO can be applied for the valuation of any type of Asian option simply through adjustment of the time period variable. Third, despite the fact that a normal probability distribution was assumed in the approximate arithmetic normal Distribution, It Did Not Generate Any Inefficient And Inaccurate Questions On Model Pricing And hedging performance. Hence, this assumption can be relaxed in future research.

However, some limitations were inevitable because, following Hull and White [1], the correlation between the instantaneous underlying price process and the stochastic volatility process was ignored. Moreover, the developed approximate analytical solution is inapplicable for derivation under the condition of mean-reversion pricing.

Author Contributions: C.-C.H. and C.-G.L. conceived of the presented idea. C.-C.H., C.-G.L. and T.-J.K. developed the theory and performed the computations. C.-C.H., C.-G.L. and T.-J.K. verified the analytical methods. C.-C.H. and C.-G.L. encouraged T.-J.K. to investigate "Pricing of Arithmetic Asian Option under Stochastic Volatility Dynamics- Overcome the Risk of High Frequent Trading" and supervised the findings of this work. All authors have read and agreed to the published version of the manuscript.

Funding: This research received no external funding.

Acknowledgments: We acknowledge any support given from Department of Business Administration in Soochow University, Taiwan, Department of Financial Engineering and Actuarial Mathematics in Soochow University, Taiwan, and Pervasive Artificial Intelligence Research (PAIR) Labs, Taiwan.

Conflicts of Interest: The authors declare no conflict of interest. 


\section{Appendix A Derivation of Hedge Ratio-Delta}

When $t \in[0, T-A]$, then

$$
\text { Delta }=\Delta=\frac{\partial f}{\partial S_{t}}=\frac{\partial C}{\partial S_{t}}+\frac{1}{2} \frac{\partial C_{2}}{\partial S_{t}} \operatorname{Var}(\bar{V})+\frac{1}{6} \frac{\partial C_{3}}{\partial S_{t}} \operatorname{Skew}(\bar{V})
$$

where

$$
\begin{aligned}
& \frac{\partial C}{\partial S_{t}}=e^{-(r-d) A-d(T-t)}\left[m_{1} N\left(\frac{m_{1}}{\sqrt{v_{1}}}\right)+\sqrt{\frac{v_{1}}{2 \pi}} e^{-\frac{m_{1}^{2}}{2 v_{1}}}\right] \\
& \frac{\partial C_{2}}{\partial S_{t}}=e^{-(r-d) A-d(T-t)}\left[M_{12} N\left(\frac{m_{1}}{\sqrt{v_{1}}}\right)+\frac{1}{\sqrt{2 \pi}} e^{-\frac{m_{1}^{2}}{2 v_{1}}} M_{11} \bar{A}_{1}-\frac{1}{\sqrt{32 \pi}} e^{-\frac{m_{1}^{2}}{2 v_{1}}} v_{1}^{-\frac{3}{2}} V_{11}^{2}\right. \\
& \left.+\frac{1}{\sqrt{8 \pi}} e^{-\frac{m_{1}^{2}}{2 v_{1}}} v_{1}^{-\frac{1}{2}} V_{11} \bar{B}_{1}+\frac{1}{\sqrt{8 \pi}} e^{-\frac{m_{1}^{2}}{2 v_{1}}} v_{1}^{-\frac{1}{2}} V_{12}\right] \\
& \operatorname{Var}(\bar{V})=\frac{2 \sigma^{4}\left(e^{k}-k-1\right)}{k^{2}}-\sigma^{4} \\
& \frac{\partial C_{3}}{\partial S_{t}}=e^{-(r-d) A-d(T-t)}\left[M_{13} N\left(\frac{m_{1}}{\sqrt{v_{1}}}\right)+\frac{1}{\sqrt{2 \pi}} e^{-\frac{m_{1}^{2}}{2 v_{1}}} \alpha_{11}+\frac{1}{\sqrt{32 \pi}} e^{-\frac{m_{1}^{2}}{2 v_{1}}} \alpha_{12}\right. \\
& \left.+\frac{1}{\sqrt{8 \pi}} e^{-\frac{m_{1}^{2}}{2 v_{1}}}\left(\alpha_{13}+\alpha_{14}\right)\right] \\
& \operatorname{Skew}(\bar{V})=\sigma^{6}\left[\frac{e^{3 k}-(9+18 k) e^{k}+\left(8+24 k+18 k^{2}-6 k^{3}\right)}{3 k^{3}}\right]
\end{aligned}
$$

in which

$$
\begin{aligned}
& m_{1}=\frac{1}{2} A(r-d)+\frac{1}{3} A^{2}(r-d)^{2}-\frac{1}{3} A^{2}(r-d) \sigma^{2}+\frac{1}{12} A^{2} \sigma^{4} \\
& v_{1}=\left[\frac{1}{3} A+\frac{3}{4} A^{2}(r-d)+\frac{7}{15} A^{3}(r-d)^{2}\right] \sigma^{2}+\left[-\frac{1}{8} A^{2}-\frac{7}{15} A^{3}(r-d)\right] \sigma^{4} \\
& +\frac{7}{60} A^{3} \sigma^{6} \\
& M_{11}=\frac{\partial m_{1}}{\partial \bar{V}}=-\frac{1}{3} A^{2}(r-d)+\frac{1}{6} A^{2} \bar{V} \\
& M_{12}=\frac{\partial^{2} m_{1}}{\partial \bar{V}^{2}}=\frac{1}{6} A^{2} \\
& M_{13}=\frac{\partial^{3} m_{1}}{\partial \bar{V}^{3}}=0 \\
& V_{11}=\frac{\partial v_{1}}{\partial \bar{V}}=\left[\frac{1}{3} A+\frac{3}{4} A^{2}(r-d)+\frac{7}{15} A^{3}(r-d)^{2}\right]+\left[-\frac{1}{4} A^{2}-\frac{14}{15} A^{3}(r-d)\right] \bar{V} \\
& +\frac{7}{20} A^{3} \bar{V}^{2} \\
& V_{12}=\frac{\partial^{2} v_{1}}{\partial \bar{V}^{2}}=\left[-\frac{1}{4} A^{2}-\frac{14}{15} A^{3}(r-d)\right]+\frac{7}{10} A^{3} \bar{V} \\
& V_{13}=\frac{\partial^{3} v_{1}}{\partial \bar{V}^{3}}=\frac{7}{10} A^{3} \\
& \bar{A}_{1}=M_{11} v_{1}^{-\frac{1}{2}}-\frac{1}{2} m_{1} v_{1}^{-\frac{3}{2}} V_{11} \\
& \bar{B}_{1}=-m_{1} v_{1}^{-1} M_{11}+\frac{1}{2} m_{1}^{2} v_{1}^{-2} V_{11}
\end{aligned}
$$




$$
\begin{gathered}
\alpha_{11}=2 M_{12} \bar{A}_{1}+M_{11} \bar{A}_{1} \bar{B}_{1}+M_{11}\left(v_{1}^{-\frac{1}{2}} M_{12}-v_{1}^{-\frac{3}{2}} M_{11} V_{11}-\frac{1}{2} m_{1} v_{1}^{-\frac{3}{2}} V_{12}+\frac{3}{4} m_{1} v_{1}^{-\frac{5}{2}} V_{11}^{2}\right) \\
\alpha_{12}=\frac{3}{2} v_{1}^{-\frac{5}{2}} V_{11}^{3}-v_{1}^{-\frac{3}{2}} V_{11}^{2} \bar{B}_{1}-2 v_{1}^{-\frac{3}{2}} V_{11} V_{12} \\
\alpha_{13}=-\frac{1}{2} v_{1}^{-\frac{3}{2}} V_{11} V_{12}+v_{1}^{-\frac{1}{2}} V_{12} \bar{B}_{1}+v_{1}^{-\frac{1}{2}} V_{13} \\
\alpha_{14}=-\frac{1}{2} v_{1}^{-\frac{3}{2}} V_{11}^{2} \bar{B}_{1}+v_{1}^{-\frac{1}{2}} V_{11} \bar{B}_{1}^{2}+v_{1}^{-\frac{1}{2}} V_{12} \bar{B}_{1} \\
+v_{1}^{-\frac{1}{2}} V_{11}\left(-v_{1}^{-1} M_{11}^{2}+2 m_{1} v_{1}^{-2} M_{11} V_{11}-m_{1} v_{1}^{-1} M_{12}-m_{1}^{2} v_{1}^{-3} V_{11}^{2}+\frac{1}{2} m_{1}^{2} v_{1}^{-2} V_{12}\right)
\end{gathered}
$$

When $t \in[T-A, T]$,

$$
\text { Delta }=\Delta=\frac{\partial f}{\partial S_{t}}=\frac{\partial C}{\partial S_{t}}+\frac{1}{2} \frac{\partial C_{2}}{\partial S_{t}} \operatorname{Var}(\bar{V})+\frac{1}{6} \frac{\partial C_{3}}{\partial S_{t}} \operatorname{Skew}(\bar{V})
$$

where

$$
\begin{aligned}
& \frac{\partial C}{\partial S_{t}}=e^{-r(T-t)}\left[m_{2} N\left(\frac{m_{2}}{\sqrt{v_{2}}}\right)+\sqrt{\frac{v_{2}}{2 \pi}} e^{-\frac{m_{2}^{2}}{2 v_{2}}}+S_{t} N\left(\frac{m_{2}}{\sqrt{v_{2}}}\right) \frac{\partial m_{2}}{\partial S_{t}}\right] \\
& \frac{\partial C_{2}}{\partial S_{t}}=e^{-r(T-t)}\left[M_{22} N\left(\frac{m_{2}}{\sqrt{v_{2}}}\right)+\frac{1}{\sqrt{2 \pi}} e^{-\frac{m_{2}^{2}}{2 v_{2}}} M_{21} \bar{A}_{2}-\frac{1}{\sqrt{32 \pi}} e^{-\frac{m_{2}^{2}}{2 v_{2}}} v_{2}^{-\frac{3}{2}} V_{21}^{2}+\frac{1}{\sqrt{8 \pi}} e^{-\frac{m_{2}^{2}}{2 v_{2}}} v_{2}^{-\frac{1}{2}} V_{22}\right. \\
& \left.+\frac{1}{\sqrt{8 \pi}} e^{-\frac{m_{2}^{2}}{2 v_{2}}} v_{2}^{-\frac{1}{2}} V_{21} \bar{B}_{2}\right]+S_{t} e^{-r(T-t)}\left[\frac{1}{\sqrt{2 \pi}} e^{-\frac{m_{2}^{2}}{2 v_{2}}} M_{22} v_{2}^{-\frac{1}{2}} \frac{\partial m_{2}}{\partial S_{t}}\right. \\
& -\frac{1}{\sqrt{2 \pi}} e^{-\frac{m_{2}^{2}}{2 v_{2}}} m_{2} v_{2}^{-1} M_{21} \bar{A}_{2} \frac{\partial m_{2}}{\partial S_{t}}+\frac{1}{\sqrt{32 \pi}} e^{-\frac{m_{2}^{2}}{2 v_{2}} m_{2} v_{2}^{-\frac{5}{2}} V_{21}^{2} \frac{\partial m_{2}}{\partial S_{t}}-\frac{1}{\sqrt{8 \pi}} e^{-\frac{m_{2}^{2}}{2 v_{2}}} m_{2} v_{2}^{-\frac{3}{2}} V_{22} \frac{\partial m_{2}}{\partial S_{t}}} \\
& \left.-\frac{1}{\sqrt{8 \pi}} e^{-\frac{m_{2}^{2}}{2 v_{2}}} m_{2} v_{2}^{-\frac{3}{2}} V_{21} \bar{B}_{2} \frac{\partial m_{2}}{\partial S_{t}}+\frac{1}{\sqrt{2 \pi}} e^{-\frac{m_{2}^{2}}{2 v_{2}}} M_{21} \frac{\partial \bar{A}_{2}}{\partial S_{t}}+\frac{1}{\sqrt{8 \pi}} e^{-\frac{m_{2}^{2}}{2 v_{2}}} v_{2}^{-\frac{1}{2}} V_{21} \frac{\partial \bar{B}_{2}}{\partial S_{t}}\right] \\
& \operatorname{Var}(\bar{V})=\frac{2 \sigma^{4}\left(e^{k}-k-1\right)}{k^{2}}-\sigma^{4} \\
& \frac{\partial C_{3}}{\partial S_{t}}=e^{-r(T-t)}\left[M_{23} N\left(\frac{m_{2}}{\sqrt{v_{2}}}\right)+\frac{1}{\sqrt{2 \pi}} e^{-\frac{m_{2}^{2}}{2 v_{2}}} \alpha_{21}+\frac{1}{\sqrt{32 \pi}} e^{-\frac{m_{2}^{2}}{2 v_{2}}} \alpha_{22}+\frac{1}{\sqrt{8 \pi}} e^{-\frac{m_{2}^{2}}{2 v_{2}}}\left(\alpha_{23}+\alpha_{24}\right)\right] \\
& +S_{t} e^{-r(T-t)}\left[\frac{1}{\sqrt{2 \pi}} e^{-\frac{m_{2}^{2}}{2 v_{2}}} M_{23} v_{2}^{-\frac{1}{2}} \frac{\partial m_{2}}{\partial S_{t}}-\frac{1}{\sqrt{2 \pi}} e^{-\frac{m_{2}^{2}}{2 v_{2}}} m_{2} v_{2}^{-1} \alpha_{21} \frac{\partial m_{2}}{\partial S_{t}}+\frac{1}{\sqrt{2 \pi}} e^{-\frac{m_{2}^{2}}{2 v_{2}}} \frac{\partial \alpha_{21}}{\partial S_{t}}\right. \\
& -\frac{1}{\sqrt{32 \pi}} e^{-\frac{m_{2}^{2}}{2 v_{2}}} m_{2} v_{2}^{-1} \alpha_{22} \frac{\partial m_{2}}{\partial S_{t}}+\frac{1}{\sqrt{32 \pi}} e^{-\frac{m_{2}^{2}}{2 v_{2}}} \frac{\partial \alpha_{22}}{\partial S_{t}}-\frac{1}{\sqrt{8 \pi}} e^{-\frac{m_{2}^{2}}{2 v_{2}}} m_{2} v_{2}^{-1}\left(\alpha_{23}+\alpha_{24}\right) \frac{\partial m_{2}}{\partial S_{t}} \\
& \left.+\frac{1}{\sqrt{8 \pi}} e^{-\frac{m_{2}^{2}}{2 v_{2}}}\left(\frac{\partial \alpha_{23}}{\partial S_{t}}+\frac{\partial \alpha_{24}}{\partial S_{t}}\right)\right] \\
& \operatorname{Skew}(\bar{V})=\sigma^{6}\left[\frac{e^{3 k}-(9+18 k) e^{k}+\left(8+24 k+18 k^{2}-6 k^{3}\right)}{3 k^{3}}\right]
\end{aligned}
$$

in which

$$
\begin{aligned}
m_{2}= & {\left[1+(T-t)(r-d)-\frac{1}{A}(T-t)-\frac{1}{2 A}(T-t)^{2}(r-d)-\frac{M_{t}}{S_{t}}\right.} \\
& \left.+\frac{1}{2}(T-t)^{2}(r-d)^{2}-\frac{1}{6 A}(T-t)^{3}(r-d)^{2}\right]+\left[-\frac{1}{2}(T-t)^{2}(r-d)\right. \\
& \left.+\frac{1}{6 A}(T-t)^{3}(r-d)\right] \sigma^{2}+\left[\frac{1}{8}(T-t)^{2}-\frac{1}{24 A}(T-t)^{3}\right] \sigma^{4}
\end{aligned}
$$




$$
\begin{aligned}
& v_{2}=\sigma^{2}(T-t)\left[1-\frac{1}{A}(T-t)+2(T-t)(r-d)+\frac{1}{3 A^{2}}(T-t)^{2}-\frac{5}{3 A}(T-t)^{2}(r-d)\right. \\
& +(T-t)^{2}(r-d)^{2}+\frac{5}{12 A^{2}}(T-t)^{3}(r-d)-\frac{2}{3 A}(T-t)^{3}(r-d)^{2} \\
& \left.+\frac{2}{15 A^{2}}(T-t)^{4}(r-d)^{2}\right]+\sigma^{4}(T-t)^{2}\left[-\frac{1}{2}+\frac{1}{2 A}(T-t)-(T-t)(r-d)\right. \\
& \left.-\frac{1}{8 A^{2}}(T-t)^{2}+\frac{2}{3 A}(T-t)^{2}(r-d)-\frac{2}{15 A^{2}}(T-t)^{3}(r-d)\right]+\sigma^{6}(T-t)^{3}\left[\frac{1}{4}\right. \\
& \left.-\frac{1}{6 A}(T-t)+\frac{1}{30 A^{2}}(T-t)^{2}\right] \\
& M_{21}=\frac{\partial m_{2}}{\partial \bar{V}}=\left[-\frac{1}{2}(T-t)^{2}(r-d)+\frac{1}{6 A}(T-t)^{3}(r-d)\right]+\left[\frac{1}{4}(T-t)^{2}\right. \\
& \left.-\frac{1}{12 A}(T-t)^{3}\right] \bar{V} \\
& M_{22}=\frac{\partial^{2} m_{2}}{\partial \bar{V}^{2}}=\left[\frac{1}{4}(T-t)^{2}-\frac{1}{12 A}(T-t)^{3}\right] \\
& M_{23}=\frac{\partial^{3} m_{2}}{\partial \bar{V}^{3}}=0 \\
& V_{21}=\frac{\partial v_{2}}{\partial \bar{V}}=\left[1-\frac{1}{A}(T-t)+2(T-t)(r-d)+\frac{1}{3 A^{2}}(T-t)^{2}-\frac{5}{3 A}(T-t)^{2}(r-d)\right. \\
& +(T-t)^{2}(r-d)^{2}+\frac{5}{12 A^{2}}(T-t)^{3}(r-d)-\frac{2}{3 A}(T-t)^{3}(r-d)^{2} \\
& \left.+\frac{2}{15 A^{2}}(T-t)^{4}(r-d)^{2}\right](T-t)+\left[-1+\frac{1}{A}(T-t)-2(T-t)(r-d)\right. \\
& \left.-\frac{1}{4 A^{2}}(T-t)^{2}+\frac{4}{3 A}(T-t)^{2}(r-d)-\frac{4}{15 A^{2}}(T-t)^{3}(r-d)\right](T-t)^{2} \bar{V} \\
& +\left[\frac{3}{4}-\frac{1}{2 A}(T-t)+\frac{1}{10 A^{2}}(T-t)^{2}\right](T-t)^{3} \bar{V}^{2} \\
& V_{22}=\frac{\partial^{2} v_{2}}{\partial \bar{V}^{2}}=\left[-1+\frac{1}{A}(T-t)-2(T-t)(r-d)-\frac{1}{4 A^{2}}(T-t)^{2}+\frac{4}{3 A}(T-t)^{2}(r-d)\right. \\
& \left.-\frac{4}{15 A^{2}}(T-t)^{3}(r-d)\right](T-t)^{2}+\left[\frac{3}{2}-\frac{1}{A}(T-t)+\frac{1}{5 A^{2}}(T-t)^{2}\right](T-t)^{3} \bar{V} \\
& V_{23}=\frac{\partial^{3} v_{2}}{\partial \bar{V}^{3}}=\left[\frac{3}{2}-\frac{1}{A}(T-t)+\frac{1}{5 A^{2}}(T-t)^{2}\right](T-t)^{3} \\
& \bar{A}_{2}=\frac{\partial}{\partial \bar{V}}\left(\frac{m_{2}}{\sqrt{v_{2}}}\right)=M_{21} v_{2}^{-\frac{1}{2}}-\frac{1}{2} m_{2} v_{2}^{-\frac{3}{2}} V_{21} \\
& \bar{B}_{2}=\frac{\partial}{\partial \bar{V}}\left(-\frac{m_{2}^{2}}{2 v_{2}}\right)=-m_{2} v_{2}^{-1} M_{21}+\frac{1}{2} m_{2}^{2} v_{2}^{-2} V_{21} \\
& \alpha_{21}=2 M_{22} \bar{A}_{2}+M_{21} \bar{A}_{2} \bar{B}_{2}+M_{21}\left(v_{2}^{-\frac{1}{2}} M_{22}-v_{2}^{-\frac{3}{2}} M_{21} V_{21}-\frac{1}{2} m_{2} v_{2}^{-\frac{3}{2}} V_{22}+\frac{3}{4} m_{2} v_{2}^{-\frac{5}{2}} V_{21}^{2}\right) \\
& \alpha_{22}=\frac{3}{2} v_{2}^{-\frac{5}{2}} V_{21}^{3}-v_{2}^{-\frac{3}{2}} V_{21}^{2} \bar{B}_{2}-2 v_{2}^{-\frac{3}{2}} V_{21} V_{22} \\
& \alpha_{23}=-\frac{1}{2} v_{2}^{-\frac{3}{2}} V_{21} V_{22}+v_{2}^{-\frac{1}{2}} V_{22} \bar{B}_{2}+v_{2}^{-\frac{1}{2}} V_{23} \\
& \alpha_{24}=-\frac{1}{2} v_{2}^{-\frac{3}{2}} V_{21}^{2} \bar{B}_{2}+v_{2}^{-\frac{1}{2}} V_{21} \bar{B}_{2}^{2}+v_{2}^{-\frac{1}{2}} V_{22} \bar{B}_{2} \\
& +v_{2}^{-\frac{1}{2}} V_{21}\left(-v_{2}^{-1} M_{21}^{2}+2 m_{2} v_{2}^{-2} M_{21} V_{21}-m_{2} v_{2}^{-1} M_{22}-m_{2}^{2} v_{2}^{-3} V_{21}^{2}+\frac{1}{2} m_{2}^{2} v_{2}^{-2} V_{22}\right) \\
& \frac{\partial m_{2}}{\partial S_{t}}=\frac{M_{t}}{S_{t}^{2}} \\
& \frac{\partial \bar{A}_{2}}{\partial S_{t}}=-\frac{1}{2} v_{2}^{-\frac{3}{2}} V_{21} \frac{\partial m_{2}}{\partial S_{t}} \\
& \frac{\partial \bar{B}_{2}}{\partial S_{t}}=-v_{2}^{-1} M_{21} \frac{\partial m_{2}}{\partial S_{t}}+m_{2} v_{2}^{-2} V_{21} \frac{\partial m_{2}}{\partial S_{t}} \\
& \frac{\partial \alpha_{21}}{\partial S_{t}}=2 M_{22} \frac{\partial \bar{A}_{2}}{\partial S_{t}}+M_{21} \bar{B}_{2} \frac{\partial \bar{A}_{2}}{\partial S_{t}}+M_{21} \bar{A}_{2} \frac{\partial \bar{B}_{2}}{\partial S_{t}}+M_{21}\left(\frac{3}{4} v_{2}^{-\frac{5}{2}} V_{21}^{2} \frac{\partial m_{2}}{\partial S_{t}}-\frac{1}{2} v_{2}^{-\frac{3}{2}} V_{22} \frac{\partial m_{2}}{\partial S_{t}}\right)
\end{aligned}
$$




$$
\begin{gathered}
\frac{\partial \alpha_{22}}{\partial S_{t}}=-v_{2}^{-\frac{3}{2}} V_{21}^{2} \frac{\partial \bar{B}_{2}}{\partial S_{t}} \\
\frac{\partial \alpha_{23}}{\partial S_{t}}=v_{2}^{-\frac{1}{2}} V_{22} \frac{\partial \bar{B}_{2}}{\partial S_{t}} \\
\frac{\partial \alpha_{24}}{\partial S_{t}}=-\frac{1}{2} v_{2}^{-\frac{3}{2}} V_{21}^{2} \frac{\partial \bar{B}_{2}}{\partial S_{t}}+2 v_{2}^{-\frac{1}{2}} V_{21} \bar{B}_{2} \frac{\partial \bar{B}_{2}}{\partial S_{t}}+v_{2}^{-\frac{1}{2}} V_{22} \frac{\partial \bar{B}_{2}}{\partial S_{t}}+v_{2}^{-\frac{1}{2}} V_{21}\left(2 v_{2}^{-2} M_{21} V_{21} \frac{\partial m_{2}}{\partial S_{t}}\right. \\
\left.-v_{2}^{-1} M_{22} \frac{\partial m_{2}}{\partial S_{t}}-2 m_{2} v_{2}^{-3} V_{21}^{2} \frac{\partial m_{2}}{\partial S_{t}}+m_{2} v_{2}^{-2} V_{22} \frac{\partial m_{2}}{\partial S_{t}}\right)
\end{gathered}
$$

\section{References}

1. Bernales, A. Make-take Decisions under High-frequency Trading Competition. J. Finance Mark. 2019, 45, 1-18. [CrossRef]

2. Shafi, K.; Latif, N.; Shad, S.A.; Idrees, Z. High-frequency trading: Inverse relationship of the financial markets. Phys. A Stat. Mech. Appl. 2019, 527, 121067. [CrossRef]

3. Bibinger, M.; Hautsch, N.; Malec, P.; Reiss, M. Estimating the Spot Covariation of Asset Prices-Statistical Theory and Empirical Evidence. J. Bus. Econ. Stat. 2019, 37, 419-435. [CrossRef]

4. Said, E.; Ayed, A.B.H.; Thillou, D.; Rabeyrin, J.-J.; Abergel, F. Market Impact: A Systematic Study of the High Frequency Options Market. Quant. Finance 2020, 21, 1-16. [CrossRef]

5. Chaboud, A.P.; Chiquoine, B.; Hjalmarson, E.; Vega, C. Rise of the Machines: Algorithmic Trading in the Foreign Exchange Market. J. Finance 2014, 69, 2045-2084. [CrossRef]

6. Baldauf, M.; Mollner, J. High-Frequency Trading and Market Performance. J. Finance 2020, 75, 1495-1523. [CrossRef]

7. Corsaro, S.; Kyriakou, I.; Marazzina, D.; Marino, Z. A general framework for pricing Asian options under stochastic volatility on parallel architectures. Eur. J. Oper. Res. 2019, 272, 1082-1095. [CrossRef]

8. Wang, X. Analytical valuation of Asian options with counterparty risk under stochastic volatility models. J. Futures Mark. 2020, 40, 410-429. [CrossRef]

9. Yao, N.; Ling, Z.; Zhang, J.; Xizo, M. Short maturity conditional Asian options in local volatility models. Math. Finance Econ. 2020, 14, 307-328. [CrossRef]

10. Heston, S.L. A Closed-Form Solution for Options with Stochastic Volatility with Applications to Bond and Currency Options. Rev. Finance Stud. 1993, 6, 327-343. [CrossRef]

11. Kemna, A.; Vorst, A. A pricing method for options based on average asset prices. J. Bank. Finance 1990, 2, $52-66$.

12. Rogers, L.C.G.; Shi, Z. The value of an Asian option. J. Appl. Probab. 1995, 32, 1077-1088. [CrossRef]

13. Hoogland, J.K.; Neumann, C.D.D. Local scale invariance and contingent claim pricing. Int. J. Theor. Appl. Finance 2001, 4, 1-21. [CrossRef]

14. Hoogland, J.K.; Neumann, C.D.D. ; Neumann, C.D.D. Local scale invariance and contingent claim pricing II. Int. J. Theor. Appl. Finance 2001, 4, 23-43. [CrossRef]

15. Vecer, J. A new PDE approach for pricing arithmetic average Asian options. J. Comput. Finance 2001, 4, 105-113. [CrossRef]

16. Vecer, J. Unified pricing of Asian options risk. J. Comput. Finance 2001, 4, 113-116.

17. Linetsky, V. Pricing Equity Derivatives Subject to Bankruptcy. Math. Finance 2006, 16, 255-282. [CrossRef]

18. Vanmaele, M.; Deelstra, G.; Liinev, J.; Dhaene, J.; Goovaerts, M.J. Bounds for the price of discrete arithmetic Asian options. J. Comput. Finance 2006, 185, 51-90. [CrossRef]

19. Hull, J.; White, A. The Pricing of Options on Assets with Stochastic Volatilities. J. Finance 1987, 42, 281-300. [CrossRef]

20. Stein, E.M.; Stein, J.C. Stock Price Distributions with Stochastic Volatility: An Analytic Approach. Rev. Financ. Stud. 1991, 4, 727-752. [CrossRef]

21. Turnbull, S.; Wakeman, L. A quick algorithm for pricing European average options. J. Financ. Quant. Anal. 1991, 26, 377-389. [CrossRef]

22. Ritchken, P.; Sankarasubramanian, L.; Vijh, A.M. The valuation of path dependent contracts on the average. Manag. Sci. 1993, 39, 1202-1213. [CrossRef] 
23. Milevsky, M.A.; Posner, S.E. Asian options, the sum of lognormals, and the reciprocal gamma distribution. J. Financ. Quant. Anal. 1998, 33, 409-422. [CrossRef]

24. Fouque, J.P.; Han, C.H. Pricing Asian options with stochastic volatility. Quant. Finance 2003, 3, $353-362$. [CrossRef]

25. Tsao, C.Y.; Chang, C.C.; Lin, C.G. Analytic Approximation Formulae for Pricing Forward Starting Asian option. J. Futures Mark. 2003, 23, 487-516. [CrossRef]

26. Sun, J.; Chen, L.; Li, S. A quasi-analytical pricing model for arithmetic Asian options. J. Futures Mark. 2013, 33, 1143-1166. [CrossRef]

27. Li, W.; Chen, S. Pricing and hedging of arithmetic Asian options via the Edgeworth series expansion approach. J. Financ. Data Sci. 2016, 2, 1-25. [CrossRef]

28. Lin, C.G.; Chang, C.C. Approximate Analytic Solution for Asian Options with Stochastic Volatility. North Am. J. Econ. Finance 2019, 54, 100949. [CrossRef]

29. Aprahamian, H.; Maddah, B. Pricing Asian options via compound gamma and orthogonal polynomials. Appl. Math. Comput. 2015, 264, 21-43. [CrossRef]

30. Dufresne, D. Laguerre series for Asian and other options. Math. Finance 1999, 10, 407-428. [CrossRef]

31. Willems, S. Asian Option Pricing with Orthogonal Polynomials. Quant. Finance 2019, 19, 605-618. [CrossRef]

32. Broadie, M.; Kaya, O. Exact Simulation of Stochastic Volatility and Other Affine Jump Diffusion Processes. Oper. Res. 2006, 54, 217-231. [CrossRef]

33. Kouritzin, M.A. Explicit Heston solutions and stochastic approximation for path-dependent option pricing. Int. J. Theor. Appl. Finance 2018, 21, 217-231. [CrossRef]

34. Kouarfate, R.; Kouritzin, A.; Mackay, A. Explicit Solution Method for the 3/2 Model; Cornell University: Ithaca, NY, USA, 2020.

35. Kirkby, J.L.; Nguyen, D. Efficient Asian option pricing under regime switching jump diffusions and stochastic volatility models. Ann. Finance 2020, 16, 307-351. [CrossRef]

Publisher's Note: MDPI stays neutral with regard to jurisdictional claims in published maps and institutional affiliations.

(C) 2020 by the authors. Licensee MDPI, Basel, Switzerland. This article is an open access article distributed under the terms and conditions of the Creative Commons Attribution (CC BY) license (http://creativecommons.org/licenses/by/4.0/). 